\title{
Activismo y criminalización de la protesta social: feunassc comunidad campesina 5 de junio de la ciudad de milagro
}

\author{
Víctor Cabezas Pinta \\ vcabezasp1@unemi.edu.ec \\ Israel Viejó Mora \\ lviejom1@unemi.edu.ec \\ Christian Castro Calle \\ christianeduardocastro@gmail.com \\ Universidad Estatal de Milagro \\ Milagro- Ecuador
}

\section{RESUMEN}

La movilización de las organizaciones sociales ante la defensa de sus derechos y de su entorno durante el gobierno de Rafael Correa fue intenso, desde la ruptura de las cercanías que mantuvieron en un momento coyuntural de política en el país, cayeron en un choque constante por los intereses de cada lado.

En este análisis se hace un recuento de la situación de la organización social Federación Única de Afiliados al Seguro Social Campesino FEUNASSC, y su activismo, movilización por la defensa de los derechos de los campesinos y comunidad agrícola, de su organización y del país. En ese marco es importante mencionar que esta organización participó junto al movimiento indígena de varias movilizaciones en contra de la política de ese gobierno. Durante el gobierno de Rafael Correa se ha pasado de una mutua aceptación y defensa a un rechazo y condena del otro. (León, 2010). Ubicando las trasgresiones realizadas desde los medios de comunicación públicos, que estaban bajo la dirección del gobierno nacional. Se busca hacer un recuento de las movilizaciones en las que participaron junto a otros colectivos y organizaciones sociales, significándoles ataques en términos despectivos por parte del mandatario ecuatoriano durante la realización de sus encuentros con la prensa.

Los resultados del proceso investigativo se plasman el análisis del contexto de la organización social y del país, la criminalización de la protesta social con el alcance de la 
comunicación estratégica como herramienta para la afectación den la imagen de las organizaciones sociales, las incidencias de procesos similares de la lucha social y reivindicativa de derechos a nivel latinoamericano.

Palabras clave: activismo; criminalización de la protesta social; gobierno; organizaciones sociales; medios de comunicación. 


\title{
Activism and criminalization of social protest: feunassc peasant community june 5 of the city of milagro
}

\begin{abstract}
The mobilization of social organizations in defense of their rights and their environment during the Rafael Correa administration was intense, since the rupture of the neighborhoods that they maintained in a temporary moment of politics in the country, fell into a constant clash for the interests of each side.

This analysis recounts the situation of the social organization Unique Federation of Affiliates to the Social Security Peasants FEUNASSC, and its activism, mobilization for the defense of the rights of farmers and agricultural community, their organization and the country. Locating the transgressions made from the public media, which were under the direction of the national government. It seeks to recount the mobilizations in which they participated together with other groups and social organizations, meaning attacks in derogatory terms by the Ecuadorian president during the conduct of his meetings with the press.

The results of the investigative process are reflected in the analysis of the context of the social organization and the country, the criminalization of social protest with the scope of strategic communication as a tool to affect the image of social organizations, the incidents of similar processes of the social struggle and demands for rights at the Latin American level.
\end{abstract}

Keywords: activism; criminalization of social protest; government: social organizations, media.

Artículo recibido: 15 noviembre. 2021 Aceptado para publicación: 10 diciembre 2021 Correspondencia: leoisraelviejo@yahoo.com Conflictos de Interés: Ninguna que declarar 


\section{INTRODUCCIÓN}

Ante el surgimiento en Ecuador de gobiernos que en su discurso argumentaban ser progresistas, nacionalistas, como el Gobierno de Lucio Gutiérrez que, durante el 2003, proclamaba una propuesta política diferente que "cambiaría el Ecuador o moriría en el intento, propuesta que no realizo y en poco tiempo huyo del país. En el contexto nacional se fue consolidando un nuevo movimiento que, desde las organizaciones sociales, y otros frentes activistas, propuso un proyecto político que plasme el anhelo de los ciudadanos de alcanzar una sociedad más justa.

Esos anhelos crecieron, se multiplicaron, a nivel nacional el discurso patriótico permitió que se sumen la mayor parte de organizaciones sociales en un solo frente y por un solo candidato, esto permitió dar una victoria contundente en las elecciones a la presidencia del país a esa nueva propuesta que lideraba el Ec. Rafael Correa Delgado, en el año 2006, vale mencionar la activa participación del movimiento indígena, que participó de manera activa con movilizaciones y también con una postura política presentaba el protagonismo de su brazo político desde 1996, cuyo referente organizativo más importante es la Confederación de Nacionalidades Indígenas del Ecuador (CONAIE), no pueda ser desconocida, la alianza social que llevó a la conformación del Movimiento de Unidad Plurinacional Pachakutik - Nuevo País (PK). (Lalander \& Ospina Peralta, 2012)

La organización social de campesinos llamada Federación Única de Afiliados al Seguro Social Campesino (FEUNASSC), realizó un continuo apoyo a las propuestas gubernamentales, planteando desde la realización de sus congresos y reuniones que era necesario aprovechar el momento político y sobre todo, apoyar la candidatura de Rafael Correa, que tenía un discurso de apego a los pequeños agricultores y campesinos, además de otras propuestas nacionalistas como la salida del país de la base militar estadounidense de Manta y de la petrolera Oxy, que operaba en el oriente ecuatoriano. Sin embargo, una vez que Correa alcanzó el poder, y luego de meses, los activistas de varias organizaciones (entre ellas FEUNASSC, PACHACUTIK), plantearon una mirada crítica a las políticas del gobierno, que no coincidían con las bases sociales.

El gobierno de turno al que apoyaron en su momento se convertiría en su confrontador, Correa al no recibir respuesta favorable a sus políticas, señaló a las organizaciones campesinas como parte de la oposición y las acusó de ser seguidoras de intereses capitalistas. El mismo gobierno que se había sentado con estas organizaciones a plantear 
políticas populares, las acusaba de conspiradoras. En poco tiempo pasaron de la visibilización, a ser acusadas de opositoras. Las propuestas de las organizaciones que no coincidían con las políticas o con el criterio del gobernante pasaron a ser señaladas como demandas sin sentido por parte del mandatario. En movilizaciones los dirigentes eran detenidos y acusados de terroristas. Dada la importancia de los medios de comunicación en la sociedad, los medios masivos de comunicación juegan un papel preponderante en la creación de opinión pública y debate público y en la generación de imaginarios sociales (Sánchez, 2010) y al tener influencia en los medios públicos, hubo mediatización contra la protesta social, activistas de movimientos sociales fueron acusados de terroristas con una campaña mediática en los medios de comunicación vinculados al estado como Ecuador Tv, Tc Televisión y Gamavisión, entre otros. Hechos parecidos a lo ocurrido en el gobierno de Jamil Mahuad, “se exacerbó la criminalización de la protesta estudiantil tanto en el discurso del gobierno como de los medios de comunicación y la represión policial alcanzó su clímax con el asesinato del joven Damián Peña en Cuenca”. (Aportes

\section{Andinos Revista electrónica de derechos humanos, 2012)}

El objetivo de este trabajo es conocer la opinión de los activistas sobre su representación mediática y, a través de su experiencia, descubrir las prácticas criminalizadoras que desplegaron los medios de comunicación para desacreditar sus demandas sociales, y que se ha mantenido durante varias décadas y gobiernos, pero también es importante mencionar lo importante que es el derecho a la manifestación, “ (...) no es posible concebir la democracia sin el derecho a manifestar libremente las opiniones" (Salazar Marín, 2010)

El estudio empírico se realizará en la comunidad campesina del recinto 5 de junio del sector Chimbo, cantón Milagro. Se entrevistará a Luis Pilalot Navarrete, Margoth Corrales, Verónica Estrella entre otros activistas de la organización social. Para la obtención de datos se aplicarán técnicas cualitativas; en concreto, se realizarán entrevistas estructuradas, siguiendo un cuestionario previo, a los principales líderes del movimiento campesino para conocer su percepción y opiniones del tratamiento mediático que recibieron sus acciones en los medios.

Partimos del supuesto de que los medios de comunicación han criminalizado las protestas campesinas, en línea con los intereses del gobierno. Este hecho habría impactado en el 
movimiento social, provocando un decaimiento en el activismo y las acciones de protesta. En la actualidad existen muchos análisis sobre la criminalización de las protestas en América Latina y el mundo, "el concepto de criminalización de la protesta ha sido usado por organismos nacionales e internacionales dedicados a la protección de los derechos humanos y por las propias organizaciones y movimientos sociales para nombrar a un conjunto de estrategias recurridas por actores estatales y no-estatales como una forma de intimidar, inhibir y deslegitimar este tipo de luchas", (Alvarado Alcázar, 2020)

\section{ESTRATEGIAS METODOLÓGICAS O MATERIALES Y MÉTODOS}

La investigación realizada tiene plena vigencia al tratarse de un tema de activismo, organizaciones sociales. El problema encontrado permite que sea de observación, no experimental.

Se aplicará una metodología de tipo cualitativa, que implica el uso de métodos que nos permitirá encontrar valiosas respuestas, se evitan datos numéricos, se registran informaciones mediante registros narrativos de los fenómenos estudiados y se participa de técnicas como las entrevistas no estructuradas y observaciones (Hernández, Fernández, \& Baptista, 2010)

De la misma manera, tendrá un abordaje de tipo analítico, debido a que busca descomponer el todo en segmentos, de forma que es posible observar las causas, la naturaleza y los efectos de un hecho particular (García \& Ortiz, 2010). la muestra ha sido seleccionada bajo el criterio probabilístico e intencional, ya que no se pretende lograr un direccionamiento general si no que se desea contar con elementos específicos que se ajusten a los fines investigativos”. (Hernández, Fernández, \& Baptista , 2015)

Además de entrevistas, se realizarán también grupos de discusión, analizado según la técnica del análisis crítico del discurso. Es importante mencionar que, para la realización de este proceso investigativo cualitativo, “(...) puede constituirse como método de apoyo en las investigaciones sobre cultura alimentaria y ser de gran ayuda para construir conocimiento y sentido en los temas explorados con esta aproximación metodológica”. (Arboleda, 2008)

\section{Para esto se seguirán los siguientes pasos:}

1. Selección y análisis de textos.

2. Elaboración de preguntas resultado del análisis de los textos, es decir el guion. 
3. Guion ante un grupo de discusión del recinto.

4. Realización del cuadro explicativo del resultado del grupo de discusión.

5. Para la recolección de datos se aplicó una técnica cualitativa de investigación, la realización de entrevistas a profundidad, siguiendo un cuestionario elaborado.

6. En concreto, se realizaron 10 entrevistas de forma presencial durante los meses de marzo y abril de 2018. Se seleccionaron informantes con dos perfiles diferentes:

\section{Estas fueron las personas entrevistadas:}

- Luis Darío Pilalot Navarrete, Presidente Nacional FEUNASSC.

- Dra. Margoth Corrales, Vicepresidenta nacional FEUNASSC

- Ramiro Vinueza, Director Quincenario Opción.

- Francisco Escandón, articulista periodista Quincenario Opción.

- Remo Cornejo Luque articulista Informativo Sembremos.

- Darwin Taco, columnista Quincenario Opción.

- Paul Jácome Segovia, articulista informativo Sembremos.

\section{Sistematización de las Entrevistas:}
A. Activismo.
B. Criminalización.
C. Política.

\section{Categoría A}

En la interpretación de las respuestas de las personas entrevistadas, nos detalla los siguientes datos:

Para las organizaciones sociales que no estaban de acuerdo a las políticas implementadas por Rafael Correa fue difícil sostener una lucha por sus derechos, el uso de los medios de comunicación públicos (Estado), fue determinante para acusarlos con términos de vagos y atrasa pueblos. Los medios de comunicación públicos fueron usados como plataforma para las acciones gubernamentales, “(...) los medios públicos, desde su creación aproximadamente en el 2008, han cumplido con el deber de informar de cada una de las obras del gobierno de Correa (...)”. (Punín Larrea, 2015)

La movilización y activismo por la defensa del Seguro Social Campesino y beneficios para el campo, pasó desapercibida para la sociedad. Las actividades que se realizan en el 
campo no tienen acogida, no han sido atendidas por los medios, lo que impide o dificulta que la sociedad conozca la razón de su activismo y lucha social.

Al no estar en la agenda de los grandes medios, y no poder dar a conocer a la sociedad sus principios organizacionales, les ha significado dificultades, pero con la tecnología y participación en informativos quincenales en formato tabloides, van llegando con información a sus comunidades. Hay un gran avance gracias al uso de las Tic “(...) Internet y las herramientas digitales, se ha amplificado el incremento de la cooperación, contribución y la reciprocidad de la sociedad civil (...)", (Galllego Trijueque \& VinaderSegura, 2019)

Con esto dan a conocer su lucha en temas de interés para el campesino, y de coyuntura nacional, lo que permite fortalecer su activismo y lucha social que desarrollan. En el contenido de estos medios esta basados en la construcción, con las organizaciones sociales estableciendo un mejor contenido social. Y a su vez permite fortalecer las relaciones de la organización social, tal como lo señala Natalia Rosana Zapata en su estudio de las Organizaciones sociales, incidencia y comunicación "visualizar actores y sus demandas, permitiendo conocer a la gente, acompañar con acciones estratégicas sus demandas y necesidades, construir y consolidar relaciones estratégicas entre sujetos e instituciones, potenciando el diálogo e intercambios entre diferentes y construyendo acuerdos" (Zapata, 2014)

\section{Categoría B}

La interpretación de los argumentos dados por los entrevistados, nos detallan que, durante el gobierno de Rafael Correa los medios de comunicación públicos fueron usados para el desprestigio de las organizaciones sociales que no estaban de acuerdo con su forma de gobernar el país.

Los espacios en estos medios de comunicación fueron usados para insultos y ataques a dirigentes, organizaciones etc., y con el objetivo de consolidar la denominada "revolución ciudadana", estos medios formaron parte del aparataje estatal de comunicación y también de su partido político Alianza País. Las pugnas se incrementaron en las movilizaciones, incluso medios aliados de los medios públicos como la televisora Telesur, informo de un incidente entre grupos de indígenas y equipo de reporteros de Ecuador TV medio de comunicación público, “el periodista, César Tacuri, acusó al dirigente de la Confederación de Pueblos de la Nacionalidad Kichwa del Ecuador o Ecuarunari, 
Carlos Pérez, de incitar a los manifestantes a "sacar de la marcha al medio de comunicación", tras calificarlos de infiltrados". (Telesur, 2015)

La comunicación de los medios vinculados al estado, establecían una mediatización ideológica- política para atacar a los opositores, y que sean mal vistos por la sociedad ecuatoriana, un ejemplo es la movilización del pueblo Saraguro que realizaron acciones reivindicativas y fueron mediatizados como incitadores a la violencia. Se realizaron ataques a la lucha social con términos como "tirapiedras, violentos", criminalizando desde los medios y llevando a la judialización a los dirigentes y organizaciones. La manipulación de la información con el objetivo de atacar a la lucha social fue un hecho durante el régimen de Correa. "Desde el inicio de su gobierno (2007-2017) el entonces presidente del Ecuador, Rafael Correa, atacó a los medios de comunicación y a los periodistas, hacia quienes no solo dirigió sus principales acometidas verbales, sino que también presentó acciones judiciales en su contra (...)”. (Stornaiolo Pimentel, 2019)

De acuerdo a un análisis realizado por la Universidad Andina Simón Bolívar, sede Ecuador, fueron decenas, los apresados por la policía con acusación directa del gobierno a quienes participaron de protestas y movilizaciones "La aprobación de una nueva Ley de Seguridad y los juicios interpuestos por el Gobierno actual, desde el inicio de este régimen, en contra de las organizaciones y movimientos sociales que se oponen a su política, han puesto nuevamente en la palestra pública al tema de la criminalización de la protesta social. Actualmente, 189 indígenas han sido demandados penalmente, principalmente por el delito de terrorismo y sabotaje; acusaciones que se han presentado en reacción a varias movilizaciones y protestas, por ejemplo, aquellas que se produjeron cuando se discutía la Ley de Minería (2009) o la Ley de Recursos Hídricos (2010), entre otras" (Universidad Andina Simón Bolivar , 2012)

Para hacer un contraste ante esta forma reaccionaria del estado para el desprestigio de las movilizaciones, las organizaciones sociales buscaron nuevos espacios para llegar a la sociedad y mostrar la realidad de sus acciones. De esta forma han ido participando dentro de quincenarios como el Opción (medio de comunicación escrito, que recoge noticias de sectores sociales y otros para sus ediciones), informativo Sembremos (que forma parte de FEUNASSC) y el uso de redes sociales.

Procurando abordar los temas de la óptica social de la organización y sus miembros, para que el resto de la sociedad pueda conocer los detalles de su movilización en el país, 
destacando tal como lo señalado en la revista Razón y Palabra "en determinadas situaciones pueden propugnar la movilización del público, en general, y de sus miembros, en particular, para proponer apoyo comunitario que permita una mejor implementación de las demandas realizadas a los poderes públicos." (Castillo, 2011)

\section{Categoría C}

El trabajo de las organizaciones sociales está vinculado a la defensa de sus derechos colectivos, al ser parte de una comunidad, de la sociedad del estado, están sujetos a las políticas gubernamentales. En todo momento se hace política debido al trabajo de organización de las personas.

La FEUNASSC ha venido proponiendo acciones en defensa del sector campesino y del Seguro Social para este sector de la sociedad, las acciones de activismo han sido para la defensa del Seguro Social, siendo el sistema de salud que protege a los campesinos del país, esa defensa, le ha costado enfrentar a los gobiernos y con Correa no fue la excepción. La organización ha planteado que debe ser mejorado este servicio con mejores medicinas y médicos, durante el gobierno de Rafael, al parecer hubo una pretensión de que este Seguro pase a ser parte del Ministerio de Salud, lo que levanto las manifestaciones de los afiliados que se opusieron a esta propuesta.

Los temas de la política económica, lucha contra la corrupción y en contra de la explotación minera, forman parte de los temas de la coyuntura nacional, por los que luchan desde su organización, y que les ha costado ser señalados como vándalos y ser encarcelados por ser luchadores sociales. Desde la realización de sus convocatorias a congresos y seminarios han buscado la difusión en los medios de comunicación, para llegar a la sociedad, demostrando su accionar y los intereses de su lucha.

Con el uso de medios de comunicación populares y comunitarios, sumados al uso de las redes sociales, buscan llegar con sus contenidos anti sistémicos, abordando temas sensibles para los campesinos, pero desde la reflexión del agricultor, y no desde el empresario el intermediario que se aprovecha sin ganar un precio justo por su trabajo. A través de los medios se busca que los gobernantes recuerden que las políticas deben ser realizadas para la gran mayoría, que son los pobres en el país, “(...) respuesta a esta atención intensa de los medios y de la gente, las organizaciones han decidido apuntarse al cambio, o, al menos, a las partes más visibles de esa transformación”. (Freire, 2009)

\section{RESULTADOS Y DISCUSIÓN}




\section{Resultado}

De acuerdo a la entrevista a profundidad realizada a los 5 dirigentes, 5 colaboradores de medios de comunicación propios o fraternos a FEUNASSC, tenemos las opiniones sobre los contenidos de información o como se ha señalado en algunos casos los medios de comunicación públicos fueron usados para promover solo la gestión gubernamental y el ataque a las organizaciones y dirigentes que opinaban las políticas que impulsaba el Ec. Rafael Correa en 10 años al mando del estado ecuatoriano.

Los argumentos de las personas coinciden en que los medios al servicio del estado fueron usados como una forma de ataque y mediatización de la protesta social, llamándolos en algunos casos violentos a los líderes, luchadores populares que se oponían al gobierno de Correa.

La cobertura de los medios de comunicación o de difusión de sus contenidos es nula, de acuerdo a las palabras de las personas entrevistadas, ellos manifiestan que su trabajo de activismo lo difunden en un medio impreso que es el Opción o el Sembremos, que actualmente está fuera de circulación por falta de recursos económicos. Por lo que procuran el uso de redes sociales o el dialogo con sus compañeros mediante las asambleas de la organización.

\section{Discusión}

Las valoraciones obtenidas por medio del proceso de investigación, por citar un ejemplo de acuerdo a las noticias de las denuncias que hace la CONAIE ante la ONU en su sitio web sobre las movilizaciones de lucha indígena. Junto con otras organizaciones sociales han denunciado al gobierno del Economista Rafael Correa, (CONAIE.ORG), detallando las formas de represión a las organizaciones que se movilizaban por sus derechos.

En cotejo con la información obtenida por medio de la herramienta de la entrevista, como una referencia de los criterios de los líderes de la organización social FEUNASSC, nos encontramos con varias definiciones sobre la cobertura de los medios de comunicación a sus movilizaciones. La mediatización de sus banderas de lucha las ha logrado por el trabajo organizativo con las personas de las comunidades, por un proceso de concienciación de los objetivos de sus luchas para beneficio de su sector y la sociedad.

Han visto la necesidad de poder poner un peso a la información que pueden difundir a la sociedad, no hay un respaldo ni difusión a sus contenidos por los grandes de medios de comunicación, ni en los medios públicos han encontrado la plataforma para la difusión 
de sus informaciones de manera honesta. Por ello han tenido la necesidad de comunicar sus actos y movilizaciones, con la realización de medios de comunicación que estén dentro de las plataformas tecnológicas y de redes sociales, tales como el Facebook. Twitter, Blogs. Además, aún poseen espacios en medios de comunicación escritos como periódicos no comerciales de educación ideológica que se distribuye entre ellos, y otro de mediana circulación en el que se difunde información de varias organizaciones sociales, como En Marcha y Opción respectivamente.

En las redes sus espacios están destinados a la difusión de la información de la organización y poder de esa manera contrarrestar la desinformación de los grandes medios o sus ataques mediáticos, sin oportunidad de poder argumentar sus defensas, "las organizaciones sociales han sabido situarse como interlocutores influyentes en el debate político y social, no de un país, sino a nivel global”. (Compolitica, 2019)

Una de las ventajas que aún mantienen es los procesos de diálogo y capacitación dentro de su organización, sin descuidar la participación en las decisiones importantes para la organización, esto les permite afirmar sus objetivos dentro de la organización e iniciar procesos formación entre sus miembros, en los que van a difundir contenidos para su fortalecimiento, “(...) por identidad nos referimos a la misión, los valores y la visión de mundo que tiene la organización (...)” (Enz, Franco, \& Spagnuolo, 2012)

\section{CONCLUSIÓN O CONSIDERACIONES FINALES}

Con este proceso de investigación realizado, se ha partido de la búsqueda de las bases teóricas como fundamento para la construcción de un análisis del caso de la organización social campesina FEUNASSC.

Con las entrevistas que sirven como fuente para el cotejo, análisis de los argumentos dados por los informantes en el proceso, de acuerdo con la metodología cualitativa, permite la comparación de los argumentos teóricos y todo lo expresado en el diálogo con los dirigentes y con las personas responsables de los medios que impulsan el trabajo de la FEUNASSC.

Encontrándose en sus argumentos varias características y criterios similares, que se había analizado con anterioridad desde el planteamiento de la investigación, como el manejo de la información y la cobertura de los medios a sus movilizaciones, entre las que cito por ejemplo: el interés del gobierno por mantener en el anonimato las banderas de lucha la movilización social, y en otros casos mediatizar de forma criminal (en las entrevistas no 
he visto mencionado ningún caso de criminalización) el activismo de varias organizaciones o grupos que no coincidan con sus políticas sociales, a pesar de estar consagrado el “(...) derecho a protestar "está protegido tanto por el derecho a la libertad de expresión como por el derecho a la libertad de reunión.” (Maldonado Ruiz, 2019)

Basados en la escaza cobertura de los medios, han logrado plantear y entregar sus propuestas a la sociedad por medios digitales que permitan a los ciudadanos conocer las propuestas del sector campesino que se agrupan en la FEUNASSC. La realización de ese evento solo tuvo cobertura por sus propios activistas y medios de comunicación de la localidad y no de los medios nacionales. Encontramos esas demandas y las opiniones de los dirigentes en la red social de videos Youtube con el título "XI Congreso Seguro Social campesino FEUNASSC fuente tvn c".

En esa constante movilización por las demandas de sus derechos encontramos otra gran movilización el 28 de agosto del 2013 en defensa del Seguro Social para los campesinos, los medios de comunicación Diario El Universo y El Comercio recogen la información, en base a esta investigación no se encontró información en medios públicos y estatales sobre esta movilización en defensa de sus intereses. "Los manifestantes pertenecen a la Federación Nacional de Afiliados a Seguro Campesino. Sus dirigentes fueron recibidos por el Procurador provincial del IESS. El funcionario aseguró que no se cerrará el Seguro Campesino". (Universo, 2013).

Para poder tener la difusión de sus informaciones a nivel nacional y poder enfrentar a los cuestionamientos sobre su propio activismo, esta organización social ha tenido que recurrir a la creación, participación y difusión de eventos, movilizaciones a través de sus redes sociales, blogs de la organización y de algunos medios cercanos a sus entornos que han dado coberturas a las participaciones que han realizado por diversos motivos.

De acuerdo a la opinión de las personas entrevistadas que son dirigentes de la organización campesina, y en base a sus argumentos que han hecho activismo desde las bases, nos permite conocer que las propuestas que generan el activismo que desarrollan desde sus trincheras, nacen del debate entre sus miembros y otras organizaciones sociales que agrupan a diversos sectores de la población del país.

Por lo tanto, están en la necesidad de poder comunicar a la sociedad sobre sus propuestas en virtud de poder de alcanzar la atención de las autoridades o gobierno de turno que pueda acoger las peticiones en los distintos temas de la justicia social que requieren las 
personas. En la formulación de la investigación se escogió a ala FEUNASSC por los 30 años de trayectoria de movilización y activismo de los derechos de los campesinos del seguro social, y de la sociedad ecuatoriana. Creada en Agosto de 1988 recoge diversos capítulos de lucha en la historia del país, por lo que se considera que la información que produce la organización debería tener la acogida por parte de los medios, evitando la distorsión y manejo inadecuado de la información que ellos producen en relación a la lucha por sus derechos. (FEUNASSC, 2009)

De acuerdo a lo analizado, y puesto en evidencia con la cita de diversas noticias en referencia a la protesta social, se evidencia que desde los medios de comunicación públicos hubo un ataque, con palabras que señalaban a los que participan de alguna movilización, como terrorista o vago. Este tipo de hechos fueron frecuentes por parte del exmandatario hacia los dirigentes y miembros de las organizaciones sociales que se oponían a sus políticas. La comunicación fue manipulada y sesgada con el objetivo de golpear a las organizaciones sociales en el país.

Luego del análisis de todo este proceso de investigación finalmente, podemos describir que la organización ha tenido que trabajar con los medios que hemos señalado anteriormente para la difusión de su activismo, trabajar por la defensa de sus derechos desde sus bases, potenciando la comunicación directa con sus miembros y activistas, permitiendo enfrentar la mediatización por parte del gobierno y de sus continuos ataques a las personas y organizaciones sociales que pensaban diferente a sus políticas sociales.

\section{LISTA DE REFERENCIAS}

Alvarado Alcázar, A. (2020). La criminalización de la protesta social: Un estado de la cuestión. Scielo, 1.5.

Aportes Andinos Revista electrónica de derechos humanos. (2012). Criminalización de la protesta social. Un recuento desde el retorno a la democracia. Aportes Andinos Revista electrónica de derechos humanos, 1-4.

Arboleda, L. M. (2008). El grupo de discusión como aproximación metodológica en investigaciones cualitativas. Redalyc.org, 2-5.

Castillo, E. A. (2011). LOS MEDIOS DE COMUNICACIÓN COMO ACTORES SOCIALES Y POLÍTICOS. Poder, Medios de Comunicación y Sociedad. RAZÓN Y PALABRA, 5-17. 
Compolitica. (22 de abril de 2019). Compolitica.com. Obtenido de Compolitica.com: https://compolitica.com/la-comunicacion-base-de-la-influencia-de-lasorganizaciones-sociales/

Enz, A., Franco, V., \& Spagnuolo, V. (2012). Manual de comunicación para organizaciones sociales: hacia una gestión estratégica y participativa. Antezanacc.com/, 2-5.

FEUNASSC. (Julio de 2009). Federación Unica Nacional de Afiliados al Seguro Social Campesino . Recuperado el 07 de Abril de 2018, de FEUNASSC: http://feunassc.blogspot.com/2009/07/

Freire, J. (8 de octubre de 2009). JuanFreire.com. Obtenido de JuanFreire.com: https://juanfreire.com/medios-sociales-y-cultura-digital-en-las-organizaciones/

Gallego Trijueque , S., \& Vinader-Segura, R. (2019). Capital social digital: las herramientas digitales como amplificadoras de la sociedad civil. Redalyc.org, 24.

Lalander, R., \& Ospina Peralta, P. (2012). Movimiento indígena y revolución ciudadana en Ecuador*. Cuestiones Políticas, 3-7.

León, T. J. (2010). Las organizaciones indígenas y el gobierno de Rafael Correa. Iconos. Revista de Ciencias Sociales, 5-8.

Maldonado Ruiz, L. M. (2019). Maldonado, L. (2019). Criminalización de la protesta social en el Ecuador, 6 (12). 65 - 77.65Criminalización de la protesta social en el Ecuador. Revista Universidad Internacional del Ecuador, 2-10.

Punín Larrea, M. I. (2015). LA ESTRATEGIA COMUNICACIONAL Y MEDIÁTICA DE RAFAEL CORREA. REDMARKA. Revista Digital de Marketing Aplicado, $2-8$.

Salazar Marín, D. (2010). El derecho a la protesta social en Ecuador.La criminalización de los manifestantes persiste pese a las amnistías. FLACSO , 101-105.

Sánchez, A. V. (2010). La acción de los medios de comunicación masiva en el Ecuador ¿Acción comunicativa o estratégica? FlacsoAndes, 3-6.

Stornaiolo Pimentel, U. (2019). Las relaciones entre Rafael Correa y la prensa ecuatoriana en el período 2006-2017: un análisis desde el discurso verbal y no verbal. Revista Digital. 
Telesur. (11 de Agosto de 2015). https://www.telesurtv.net/. Obtenido de https://www.telesurtv.net/: https://www.telesurtv.net/news/Indigenas-opositoresagreden-a-equipo-de-Ecuador-TV-20150811-0001.html

Universidad Andina Simón Bolivar . (2012). Criminalización de la protesta social. Un recuento desde el retorno a la democracia. Revista electrónica de derechos humanos, 1-5.

Universo, E. (28 de Agosto de 2013). eluniversocom. Recuperado el 07 de abril de 2018, de youtube: https://www.youtube.com/watch?v=00DvrIQTi_M

Zapata, N. R. (2014). ORGANIZACIONES SOCIALES, INCIDENCIA Y COMUNICACIÓN. Universidad Nacional de Quilmes , 4-8. 